\title{
Autacoid 14S,21R-Dihydroxy-Docosahexaenoic Acid Counteracts Diabetic Impairment of Macrophage Prohealing Functions
}

\author{
Haibin Tian, Yan Lu, Shraddha P. Shah, and \\ Song Hong \\ From the Center of Neuroscience Excellence, Louisiana State \\ University Health Sciences Center, New Orleans, Louisiana
}

Impaired macrophage functions imposed by diabetic complications and the suppressed formation of $14 \mathrm{~S}$, $21 R$ - dih ydroxydocos a - 4Z, 7Z, 10Z, 12E, 16Z, $19 Z$-hexaenoic acid $(14 S, 21 R$-diHDHA) in wounds contribute significantly to deficient wound healing in diabetics, but how are macrophage functions and $14 S, 21 R$-diHDHA formation associated? We studied $14 S, 21 R$-diHDHA generation from macrophages using liquid chromatography/mass spectrometry. The role in macrophage-mediated wound healing functions was determined using a murine splinted excisional wound healing model and in vitro assays. $14 \mathrm{~S}, 21 \mathrm{R}$ diHDHA acts as a macrophage-generated autacoid, and its attenuated formation in macrophages of diabetic $d b / d b$ mice was accompanied by impairment of macrophage prohealing functions. $14 S, 21 R$-diHDHA restored $d b / d b$ macrophage-impaired prohealing functions by promoting wound re-epithelialization, formulation of granulation tissue, and vascularization. Additionally, 12/15-lipoxygenase-deficient macrophages, which are unable to produce $14 S, 21 R$-diHDHA, exhibited impaired prohealing functions, which also were restored by $14 S, 21 R$ diHDHA treatment. The molecular mechanism for $14 S, 21 R$-diHDHA-induced recovery of impaired prohealing functions of $d b / d b$ macrophages involves enhancing their secretion of vascular endothelial growth factor and platelet-derived growth factor BB, decreasing hyperglycemia-induced generation of reactive oxygen species, and increasing IL-10 expression under inflammatory stimulation. Taken together, these results indicate that deficiency of $14 S, 21 R$-diHDHA formation by diabetic macrophages contributes to their impaired prohealing functions. Our findings provide mechanistic insights into wound healing in diabetics and suggest the possibility of using autologous macrophages/monocytes, treated with $14 S, 21 R$-diHDHA, or related compounds, to promote diabetes-impaired wound healing. (Am J Patbol 2011, 179:1780-1791; DOI: 10.1016/j.ajpath.2011.06.026)

Impaired wound healing associated with diabetes mellitus presents a major unresolved medical challenge. It is predicted that dysregulated cellular and molecular processes are responsible for impairments in wound healing in diabetes, ${ }^{1}$ but the mechanisms underlying these processes require further elucidation. Macrophages play critical roles in wound healing: they engulf apoptotic polymorphonuclear leukocytes (PMNs), tissue debris, and infectious microorganisms; they promote wound healing, including accelerating re-epithelialization by promoting keratinocyte migration from the wound edge to decrease the epithelial gap and increasing the formation of granulation tissue by promoting fibroblasts and endothelial cells moving into wounds to cover the wound bed ${ }^{2}$; and they enhance angiogenesis, to repair damaged blood vessels and thus to supply sufficient oxygen and nutrients during healing. ${ }^{2}$ Macrophage prohealing functions have been confirmed: macrophage transplantation into wounds promotes healing, ${ }^{3,4}$ and macrophage depletion results in delayed wound healing. ${ }^{5,6}$ The presence of diabetes, however, compromises macrophage functions and so contributes to disrupted wound healing. ${ }^{1,4,7}$

In addition to amino acid-based cytokines and growth factors, which are known to mediate wound healing and associated angiogenesis, lipid-derived molecules also may be important mediators. Docosahexaenoic acid $(\mathrm{DHA})$, an essential $\omega$-3 fatty acid, is a relatively abun-

Supported by NIH grant 1-R01-DK087800 (S.H.) and start-up funds from the Neuroscience Center of Excellence, LSUHSC-NO (S.H.).

Accepted for publication June 14, 2011.

H.T. and Y.L. contributed equally to the present work.

Supplemental material for this article can be found at http://ajp. amjpathol.org or at doi: 10.1016/j.ajpath.2011.06.026.

Address reprint requests to Song Hong, Ph.D., LSUHSC-Center of Neuroscience Excellence, Lions Building, 2020 Gravier St., Suite D, New Orleans, LA 70112. E-mail: shong@|suhsc.edu. 
dant, endogenous lipid component in blood and in wounded full-thickness skin. ${ }^{8}$ Previous studies showed that DHA can ameliorate diabetes complications, such as cardiovascular disease, by improving endothelial function through anti-inflammatory mechanisms and reduced platelet aggregation, as well as by reducing blood triglyceride levels and increasing high-density lipoprotein cholesterol. ${ }^{9-11} \mathrm{DHA}$ can also significantly promote wound healing by enhancing angiogenesis. ${ }^{12}$ Resolvins, neuroprotectins/protectins, and maresins are lipid mediators generated naturally from DHA during acute inflammation that have potent anti-inflammatory and protective functions. ${ }^{13,14}$ Their discovery has opened a new avenue for unraveling the mechanisms behind the beneficial effects of DHA. The DHA-derived lipid mediator neuroprotectin D1 (NPD1; 10R,17S-dihydroxydocosa-4Z,7Z,11E,13E,15Z,19Z-hexaenoic acid) enhances corneal wound healing, ${ }^{15}$ and $14 S, 21 R$-dihydroxydocosa-4Z,7Z,10Z,12E,16Z,19Z-hexaenoic acid (14S,21RdiHDHA), a novel endogenous DHA-derived lipid mediator, is generated by macrophages and in wounded skin. ${ }^{8,16} 14 S, 21 R$-diHDHA is produced by sequential enzymatic actions of 14S-hydroperoxylation-specific leukocyte-12-lipoxygenase (L-12-LOX) and $\omega$-1-hydroxylation-specific P450. ${ }^{8}$ When applied to wounds, $14 S, 21 R$-diHDHA potently promotes healing and angiogenesis in wounds. ${ }^{8,16}$

Diabetes and the accompanying hyperglycemia and oxidative stress damage DNA, proteins, and lipids in various tissues. These conditions cause cellular and enzymatic dysfunction during the formation of cytokines and eicosanoids associated with cell growth, angiogenesis, and/or inflammation, ${ }^{4,7,17-19}$ all of which are at least in part responsible for impairment of macrophage functions in diabetes. ${ }^{18,19}$ We have observed that $14 S, 21 R$ diHDHA formation was significantly halted in diabetic wounds, compared with nondiabetic wounds in mice, which suggests that diabetic complications dysregulate the generation of DHA-derived lipid mediators. ${ }^{16}$ In addition, supplementation of $14 S, 21 R$-diHDHA into diabetic wounds counteracts diabetes-associated impairment of wound healing, ${ }^{16}$ indicating that $14 S, 21 R$-diHDHA is an important prohealing lipid mediator. Also, decreased 14S,21R-diHDHA formation in diabetes may affect prohealing functions of cells that participate in wound healing.

The findings presented here demonstrate that diabetes-impaired prohealing functions of $d b / d b$ macrophages are associated with decreased formation of 14S,21RdiHDHA, and could be ameliorated by treating diabetic macrophages with 14S,21R-diHDHA.

\section{Materials and Methods}

\section{Mice}

Animal protocols were approved by the Institutional Animal Care and Use Committee and the Institutional Review Board of the Louisiana State University Health Sciences Center, New Orleans. Studies were blinded. Diabetic $d b / d b$ (BKS.Cg-m $+/+L e p r^{d b}$ ) and nondiabetic $d b /+$ mice (10-week-old females; Jackson Laboratory, Bar Harbor, ME) were used when blood glucose was 22 to 35 $\mathrm{mmol} / \mathrm{L}$ for $d b / d b$ mice and 5 to $10 \mathrm{mmol} / \mathrm{L}$ for $d b /+$ mice, as measured with a glucometer. Both B6.129S2Alox15 tm1Fun/J [12/15 (or leukocyte-12)-lipoxygenase deficient $\left(12 / 15-\mathrm{LOX}^{--}\right)$] and wild-type control (C57BL/6J) mice were 10-week-old females obtained from the Jackson Laboratory.

\section{Cell Cultures}

Macrophages were isolated and identified as previously described. ${ }^{4,5}$ Briefly, mice were treated with thioglycolate for 3 days. Macrophages were obtained by peritoneal lavage and were cultured in RPMI 1640 medium (Invitrogen, Carlsbad, CA) containing glucose at different concentrations; nonadherent cells were deleted by changing the culture medium. For identification of macrophages, cells were seeded into eight-well culture slides (BD Biosciences, San Jose, CA). After being fixed in $4 \%$ paraformaldehyde (Sigma-Aldrich, St. Louis, MO) for 15 minutes and permeabilized in $0.1 \%$ Triton- 100 (Sigma-Aldrich) on ice for 5 minutes, cells were incubated with primary antibody (monoclonal rat anti-mouse F4/80; Santa Cruz Biotechnology, Santa Cruz, CA) at $4^{\circ} \mathrm{C}$ for 12 hours. Cells were then incubated with Cy3-labeled goat anti-rat IgG (Invitrogen) at $4^{\circ} \mathrm{C}$ for 12 hours. Nuclei were stained with Hoechst 33342 dye (blue). Images were captured using a Zeiss Axioplan 2 microscope in an Everest digital microscopy workstation with associated software (Slidebook version 4.2; Zeiss). More than 95\% of cells were $\mathrm{F} 4 / 80^{+}$(see Supplemental Figure S1A at http://ajp.amjpathol.org).

Murine fibroblast cell line CF-1 was obtained from the American Type Culture Collection (ATCC, Manassas, $V A$ ), and cultured in Dulbecco's modified Eagle's medium containing 10\% fetal bovine serum. Dermal microvascular endothelial cells (DMVECs) were isolated from skin of $d b / d b$ mice and confirmed by immunocytochemical analysis, which demonstrated their purity (>95\%) based on the staining of endothelial markers CD31 (red) and VE-cadherin (green); nuclei were stained with Hoechst 33342 blue dye (see Supplemental Figure S1B at $h$ ttp://ajp.amjpathol.org). ${ }^{16}$ Human peripheral blood provided by the New Orleans Blood Bank was collected from a code-deidentified healthy donor who had not taken medication for 2 weeks before donation. Blood was also collected by cardiac puncture from C57BL/6J mice under deep anesthesia.

Polymorphonuclear leukocytes and platelets were separated from human or murine blood as described previously. ${ }^{20,21}$ Briefly, peripheral blood samples were mixed with the same volume of PBS containing $4 \%$ dextran and were centrifuged at $150 \times g$ for 15 minutes. Platelet-rich supernatant was further centrifuged at $1600 \times g$ for 15 minutes to collect platelets. Cell deposit was used to isolate PMNs by density gradient centrifugation using Histopaque 1.077 cell separation medium (Sigma-Aldrich). PMNs had at least 95\% purity by Hoechst 33342 dye staining and 95\% viability by Trypan Blue dye (Sigma- 
Aldrich) exclusion. Lymphocytes were isolated from the spleens of $d b /+$ mice by magnet-based microbead CD45R (B cells) and CD90.2 (T cells) according to the manufacturer's protocol (Miltenyi Biotec, Bergisch Gladbach, Germany). Lymphocytes showed more than 95\% viability by Trypan Blue dye exclusion.

\section{Formation of 14S,21R-diHDHA and Its Biosynthetic Intermediates and Analysis by Liquid Chromatography-Ultra Violet Detector- Tandem Mass Spectrometry}

The formation of $14 S, 21 R$-diHDHA and its biosynthetic intermediates and analysis by liquid chromatographytandem mass spectrometry were performed as described previously, 8,16 with some modifications. DHA, $\mathrm{DHA}-\mathrm{d}_{5}$, and porcine L-12-LOX were supplied by Cayman Chemical (Ann Arbor, MI). Neuroprotectin/protectin D1 (10R,17S-dihydroxy-docosa-4Z,7Z,11E,13E,15Z,19Zhexaenoic acid) prepared by total organic synthesis was kindly provided by Dr. Charles N. Serhan (Harvard Medical School) ${ }^{14,22}$ and Dr. Nicolas G. Bazan (Neuroscience Center of Excellence, Louisiana State University Health Sciences Center, New Orleans). ${ }^{23}$ Samples and standards of lipid mediators were handled under dimmed light, stored at $-80^{\circ} \mathrm{C}$ in vials purged with nitrogen gas and sealed tightly. Aqueous solutions of DHA and DHA derivatives were freshly prepared $\left(\sim 4^{\circ} \mathrm{C}\right)$ immediately before use.

The separation and analysis of DHA-derived compounds were performed using the liquid chromatography-photodiode array ultra violet detector-linear ion trap mass spectrometer (LC-UV-MS/MS). The LC system was equipped with a chiral column. The mobile phase had a flow-rate of $0.2 \mathrm{~mL} / \mathrm{min}$, eluted as $\mathrm{B}$ (methanol: $\mathrm{H}_{2} \mathrm{O}$ : acetic acid $=27: 73: 0.01$ ) from 0 to 1 minute, ramped from B:methanol $40: 60$ to B:methanol $20: 80$ by 50 minutes, ramped to $100 \%$ methanol by 55 minutes, and then maintained in $100 \%$ methanol. An isotope dilution LCMS/MS method was used to quantify $14 S, 21 R$-diHDHA or mono-HDHAs with $14 S, 21 R$-diHDHA-d ${ }_{4}$ or $14 S$-hydroxyDHA- $d_{5}\left(14 S-H D H A-d_{5}\right)$ as the internal standard, respectively, which was added to each macrophage sample at the time of collection (10 $\mathrm{ng}$ of each standard). ${ }^{24-28}$ The UV spectrum was acquired without an internal standard. 14S-HDHA, $14 S-H D H A-d_{5}, 14 S, 21 R-d i H D H A$, or $14 S, 21 R-$ diHDHA- $d_{4}$ from the effluent of the chiral LC coupled to the mass spectrometer was analyzed on the basis of selected MS/MS ion chromatograms at a mass-to-charge ratio $(\mathrm{m} / \mathrm{z}) 205$ of MS/MS 343, m/z 205 of MS/MS 348, m/z 253 of MS/MS 359, or $\mathrm{m} / z 253$ of MS/MS 363, respectively. The amount of $14 S-H D H A$ or $14 S, 21 R$-diHDHA in each macrophage sample was then quantified based on its LC peak area ratio relative to that of its deuterated internal standard $14 S-H D H A-d_{5}$ or $14 S, 21 R-d i H D H A-d_{4}$, respectively, using a calibration curve obtained from a series of diluted standards using stable isotope dilution LC-MS/MS method. ${ }^{24-28}$

14S,21R-diHDHA and 14S,21S-diHDHA or their deuterated isotopomers were generated by incubating $14 S$ -
HDHA $(50 \mu \mathrm{g})$ or $14 \mathrm{~S}-\mathrm{HDHA}-\mathrm{d}_{5}(50 \mu \mathrm{g})$, respectively, with 1 nmol human cytochrome P450 kit (h-P450, containing recombinant human CYP2E1, NADPH cofactor, and buffer salts in the company's proprietary ratios; Codexis-BioCatalytics, Pasadena, $\mathrm{CA})\left(30^{\circ} \mathrm{C}, 12\right.$ hours). Under the same conditions, 14R,21R-diHDHA and 14R,21SdiHDHA were generated from $14 R-\mathrm{HDHA}$ by h-P450. ${ }^{8}$ 14S-HDHA or $14 S-H D H A-d_{5}$ were prepared by incubating DHA or DHA- $d_{5}(50 \mu \mathrm{g}$ each), respectively, with porcine L-12-LOX (1000 U) in buffer containing $0.1 \mathrm{~mol} / \mathrm{L}$ Tris at $\mathrm{pH} 7.4$ for 20 minutes at $37^{\circ} \mathrm{C}$. At the end of the incubation, $3 \mathrm{mg}$ of $\mathrm{NaBH}_{4}$ was added. ${ }^{8,13}$ Additionally 14S-HDHA and 14R-HDHA were prepared from racemic 14-S/R-HDHAs (Cayman Chemical) by the above chiral LC-UV-MS/MS-based separation. The DHA and 14SHDHA used to make 14S-HDHA and 14S,21R-diHDHA, respectively, were not less than $98 \%$ pure (without detectable oxidation artifacts), as analyzed by chiral LC-UV$\mathrm{MS} / \mathrm{MS}$

The biosynthesis of 14S,21R-diHDHA and intermediates was assayed by incubating (20 minutes) purified macrophages, PMNs, lymphocytes ( $T$ cells/B cells 1:1), fibroblast cells, or DMVECs $\left(1 \times 10^{7}\right.$ cells/each type $)$, as well as platelets $\left(1 \times 10^{8}\right.$ cells), in PBS containing DHA (3 $\mu \mathrm{mol} / \mathrm{L})$ or $14 \mathrm{~S}-\mathrm{HDHA}-\mathrm{d}_{5}(1 \mu \mathrm{mol} / \mathrm{L})$; this was followed by stimulating the cells $\left(37^{\circ} \mathrm{C}, 1\right.$ hour $)$ with TNF $\alpha(10 \mathrm{ng} / \mathrm{mL})$, $\mathrm{IL}-1 \beta(10 \mathrm{ng} / \mathrm{mL})$, and lipopolysaccharide (100 ng/mL). LC-MS/MS analysis indicated that DHA (free acid) in fullthickness murine skin wounds can reach levels as high as 1 to $9 \mu \mathrm{mol} / \mathrm{kg}$. Thus, $3 \mu \mathrm{mol} / \mathrm{L} \mathrm{DHA}$ is within the concentration range found in the pathophysiological condition of skin wounds. TNF $\alpha$ and IL-1 $\beta$ are generated in tissue injury. LPS treatment simulates the potential for infection in wounds. ${ }^{8,11,29} \mathrm{TNF} \alpha, \mathrm{IL}-1 \beta$, and LPS increase the expression of phospholipase $\mathrm{A} 2$, which releases DHA free acid from membranes and/or other parts of the cells. They also likely enhance 12/15-lipoxygenase-like activity, ${ }^{26}$ which results in 14-hydroxylation of DHA and some DHA derivatives. ${ }^{20}$

Each incubation was extracted for analysis and preparative separation of $14 S, 21 R$-diHDHA or its biosynthetic intermediates. The $\mathrm{pH}$ of each incubation was adjusted to $\mathrm{pH} 4.5$ with $\mathrm{HCl}(1 \mathrm{~mol} / \mathrm{L}$, on ice) and then the incubation was mixed with three volumes of ice-cold methanol (sample:methanol $=1: 3 \mathrm{v} / \mathrm{v}$ ). Each mixture was sonicated and centrifuged $(4500 \times \mathrm{g}$ for 15 minutes at $\sim 4^{\circ} \mathrm{C}$ ). The pellet was extracted with methanol twice. The supernatants for each sample were pooled together and adjusted to $10 \%$ methanol with water, and partially purified via C18 solid-phase extraction (500 mg Bond Elut LRC-C18 INT sorbent; Agilent Technologies, Santa Clara, CA). The methanol contained $0.1 \%$ butylated hydroxytoluene to suppress auto-oxidation. ${ }^{28}$ The final extracts were reconstituted into methanol for chiral LC-UV-MS/MS separation or analysis. For chiral LC preparative separation and fraction collection of compounds generated from incubation, a fraction (10\%) of LC flow was split to MS/MS for detection. The fraction of 14S,21R-diHDHA was dried completely with $\mathrm{N}_{2}$ gas and reconstituted in ethanol. The purified 14S,21R-diHDHA and 14S,21R-diHDHA-d ${ }_{4}$ were found to be not less than $95 \%$ pure by LC-UV-MS/MS 
using neuroprotectin/protectin-D1 ${ }^{27}$ as a standard. Additionally, the purified $14 S-H D H A-d_{5}$ was quantified by LCUV-MS/MS using 14-HDHA (Cayman Chemical) as the standard.

\section{Splinted Excisional Wound Healing Model}

This model was established as previously described. ${ }^{16,30,31}$ Briefly, paired 5-mm circular, full-thickness wounds were made symmetrically along the midline on the dorsal skin of mice. Two days later, 14S,21R-diHDHA (50 ng/wound) or macrophages and 14S,21R-diHDHAtreated macrophages ( $10^{6}$ cells/wound) were applied to the wound bed (10 $\mu \mathrm{L} / \mathrm{bed})$ and were injected intradermally at four points ( $10 \mu \mathrm{L} / \mathrm{site})$ distributed evenly near the wound edge (50 $\mu \mathrm{L}$ total/wound). A donut-shaped silicone splint was adhered around the wound to minimize wound contraction; the splinting did not influence re-epithelialization. ${ }^{30}$ This model closely parallels human wound healing. The adherent macrophages were incubated with $100 \mathrm{nmol} / \mathrm{L} 14 \mathrm{~S}, 21 R$-diHDHA in a dish $\left(37^{\circ} \mathrm{C}\right.$, $5 \% \mathrm{CO}_{2}, 24$ hours), resuspended in RPMI 1640 medium, and used as 14S,21R-diHDHA-treated macrophages.

\section{Wound Healing Analysis}

Complete re-epithelialization takes $\sim 10$ days in wounds of $d b / d b$ mice and $\sim 7$ days in C57BL/6J mice. ${ }^{16,31,32}$ To enable quantification of the healing parameters appropriate for our research, re-epithelialization, granulation tissue formation, and angiogenesis were analyzed for wounds at different times for different mice. ${ }^{16}$ Briefly, wounds rimmed with $2 \mathrm{~mm}$ of unwounded skin were excised from sacrificed mice. Cryosections (10 $\mu \mathrm{m}$ thick) of wounds from $d b / d b$ mice (day 8 after wounding) and 12/15-LOX ${ }^{-1-}$ or C57BL/6J mice (day 5 after wounding) were analyzed to determine the epithelial gap and granulation tissue formation after H\&E staining. The splint has a constant area, and it was used to normalize the wound sizes $^{30}$; therefore, the original epithelial gap (original wound size) was equal in each wound $(5 \mathrm{~mm}$ in diameter).

The advancing epithelial gap is defined as the distance between the advancing edges of migrated keratinocytes. ${ }^{30,33}$ The relative epithelial gap was calculated as a percentage: (advancing epithelial gap/original epithelial gap) $\times 100$. Granulation tissue locates between epidermal layer and subcutaneous layer, and exhibits tight granular morphology. Relative granulation tissue area was calculated as a percentage: (granulation tissue area in treatment/granulation tissue area in control) $\times$ 100. Wound vascularity in wounds from $d b / d b$ mice (day 8 after wounding) and 12/15-LOX ${ }^{-1-}$ or C57BL/6J mice (day 7 after wounding) were assessed by staining vessel endothelial cells with monoclonal rat anti-mouse CD31 antibody followed by goat anti-rat IgG horseradish peroxidase detection kit (BD Biosciences, San Jose, CA), and then by hematoxylin for nuclei. Three to four images per section were captured. Percentage vascularity was calculated as $\left(\mathrm{CD} 31^{+}\right.$area per field/total wound bed area per field) $\times 100$. Images were captured on a micro- scope (Zeiss Axio Imager.M1) with an AxioCam MRC camera and Axiovision release 4.6 software. Distance of the epithelial gap, granulation tissue area, and CD31+ area were measured using ImageJ version 1.40 software $(\mathrm{NIH}$, Bethesda, MD).

\section{Macrophage-Conditioned Medium Preparation}

Quiescent $d b / d b$ and $d b /+$ macrophages $\left(3 \times 10^{5}\right.$ cells) were prepared by incubating cells in RPMI 1640 medium containing $25 \mathrm{mmol} / \mathrm{L}$ glucose (for 24 hours), and then in fresh RPMI 1640 medium containing $25 \mathrm{mmol} / \mathrm{L}$ glucose with or without $100 \mathrm{nmol} / \mathrm{L}$ 14S,21R-diHDHA (for 24 hours). The end product, macrophage-conditioned medium $(\mathrm{MCM})$, was collected after centrifugation $(3000 \times g$ for 15 minutes at $\left.4^{\circ} \mathrm{C}\right)$.

\section{DMVEC Migration and Vasculature Formation}

Analysis of DMVEC migration and vasculature formation was performed as described previously. ${ }^{16,32}$ Briefly, quiescent $d b / d b$ DMVECs were resuspended in RPMI 1640 medium with $25 \mathrm{mmol} / \mathrm{L}$ glucose and added to the upper chamber of 24-well Transwell supports (8- $\mu \mathrm{m}$ pore; BD Biosciences) at $1 \times 10^{5}$ cells/well; RPMI 1640 medium with $25 \mathrm{mmol} / \mathrm{L}$ glucose or MCM was added to the lower chamber for migration $\left(37^{\circ} \mathrm{C}, 5 \% \mathrm{CO}_{2}, 4\right.$ hours). For vasculature formation, quiescent $d b / d b$ DMVECs were cultured on Matrigel substrate in a 96-well culture plate (BD Biosciences) at $2 \times 10^{4}$ cells/well in RPMI 1640 medium with $25 \mathrm{mmol} / \mathrm{L}$ glucose or $\mathrm{MCM}\left(37^{\circ} \mathrm{C}, 5 \% \mathrm{CO}_{2}\right.$, 24 hours). Images were captured on a microscope (Nikon TS100) with a CCD camera and software (NISElements $F$ version 2.30). The vasculature length was measured using $\mathrm{NIH}$ ImageJ version 1.40 software.

\section{Bio-Plex Protein Array and Enzyme-Linked Immunosorbent Assay}

Vascular endothelial growth factor (VEGF) and plateletderived growth factor BB (PDGF-BB) secreted by macrophages treated with or without 14S,21R-diHDHA for 24 hours were quantified using a Bio-Plex kit (Bio-Rad, Hercules, CA). In another experiment, quiescent $d b / d b$ macrophages were treated with $14 S, 21 R$-diHDHA with or without LPS for 24 hours. IL-10 in the supernatants was quantified using an enzyme-linked immunosorbent assay kit (Fisher Scientific, Rockford, IL).

\section{Western Blot Analysis}

The Western blot experiments were conducted as previously described. ${ }^{16,34,35}$ Briefly, The $d b / d b, d b /+, 12 / 15-$ $\mathrm{LOX}^{-1-}$ and C57BL/6J peritoneal macrophages were analyzed for 12/15-LOX expression. In another experiment, quiescent macrophages were cultured in RPMl 1640 medium containing $5.5 \mathrm{mmol} / \mathrm{L}$ glucose (for $\mathrm{db} /+, 12 / 15$ $\mathrm{LOX}^{-1-}$, and C57BL/6J) or $25 \mathrm{mmol} / \mathrm{L}$ glucose (for $\mathrm{db} / \mathrm{db}$ ) with or without 14S,21R-diHDHA and/or IL-4 for 24 hours. Macrophages were analyzed for arginase-1 expres- 
sion. ${ }^{36}$ Western blot analysis was performed by loading $30 \mu \mathrm{g}$ total protein/lysed sample to a well in a $4 \%$ to $15 \%$ Tris-HCI SDS-PAGE gel (Bio-Rad). The electrotransferred protein bands were stained by primary antibodies and then by fluorescent-labeled secondary antibodies (LI$\mathrm{COR}$, Lincoln, NE), and finally were quantified using a LI-COR Odyssey imaging system.

\section{Reactive Oxygen Species Generation Analysis in Macrophages}

Analysis of reactive oxygen species (ROS) generation was performed as described previously. ${ }^{37}$ Briefly, The $d b / d b$ and $d b /+$ macrophages were cultured in RPMI 1640 medium containing $0.1 \%$ fetal bovine serum and 5.5 or 25 $\mathrm{mmol} / \mathrm{L}$ glucose, with or without $100 \mathrm{nmol} / \mathrm{L} 14 S, 21 R$ diHDHA, for 3 days. Medium was changed every day. ROS generation in cells was assessed using the probe 2,7-dichlorofluorescein diacetate (DCFH-DA, $10 \mu \mathrm{mol} / \mathrm{L}$; Sigma-Aldrich). Cells were cultured with DCFH-DA in RPMI 1640 medium for 30 minutes and DCFH-DA fluorescence was measured using an excitation wavelength of $480 \mathrm{~nm}$ and an emission wavelength of $535 \mathrm{~nm}$.

\section{Statistical Analysis}

Statistical analysis was performed using one-way analysis of variance followed by Fisher's least significant difference post hoc comparison. $P<0.05$ was considered significant.

\section{Results}

\section{S,21R-diHDHA Formation Is Decreased in Macrophages of Diabetic $\mathrm{db} / \mathrm{db}$ Mice}

To determine effects of diabetes on macrophage production of the prohealing, DHA-derived lipid mediator $14 S, 21 R$-diHDHA ${ }^{8}$ and the biosynthetic mechanism, we analyzed 14S,21R-diHDHA from murine macrophages using a LC-UV-MS/MS (Thermo Fisher Scientific, Waltham, MA) equipped with a chiral column (Chiralpak-IA, $150 \mathrm{~mm} \times 2.1 \mathrm{~mm} \times 5 \mu \mathrm{m}$; Chiral Technologies, West Chester, PA). Murine macrophages were isolated from the peritoneal cavity, and $14 S, 21 R$-diHDHA was generated by macrophages in both $d b / d b$ and $d b /+$ mice (Figure 1). The tandem MS/MS spectrum of peak III (Figure $1 \mathrm{~A}$ ) possessed ions at $\mathrm{m} / \mathrm{z} 359[\mathrm{M}-\mathrm{H}]^{-}, 341[\mathrm{M}-\mathrm{H}$ $\left.\mathrm{H}_{2} \mathrm{O}\right]^{-}, 323\left[\mathrm{M}-\mathrm{H}-2 \mathrm{H}_{2} \mathrm{O}\right]^{-}, 297\left[\mathrm{M}-\mathrm{H}-\mathrm{CO}_{2}-\mathrm{H}_{2} \mathrm{O}\right]^{-}$, and $279\left[\mathrm{M}-\mathrm{H}-\mathrm{CO}_{2}-2 \mathrm{H}_{2} \mathrm{O}\right]^{-}$, demonstrating two hydroxyl groups and one carboxyl group in the molecular ion $\mathrm{m} / \mathrm{z}$ 359. The 14-hydroxyl was demonstrated by ions $\mathrm{m} / \mathrm{z} 205$, 233,189 , and 161 . The 21-hydroxyl was demonstrated by ions $\mathrm{m} / \mathrm{z} 271$ and 253, which involved the cleavage of C20-C21 bond in the molecular ion at $\mathrm{m} / \mathrm{z} 359[\mathrm{M}-\mathrm{H}]^{-}$ and loss of $\mathrm{CH}(\mathrm{O}) \mathrm{CH}_{3}$ (44 atomic units) derived from the terminal group of carbons at the tail of the molecular ion (Figure 1, A and B). Therefore, the peak III compound was 14,21-diHDHA.

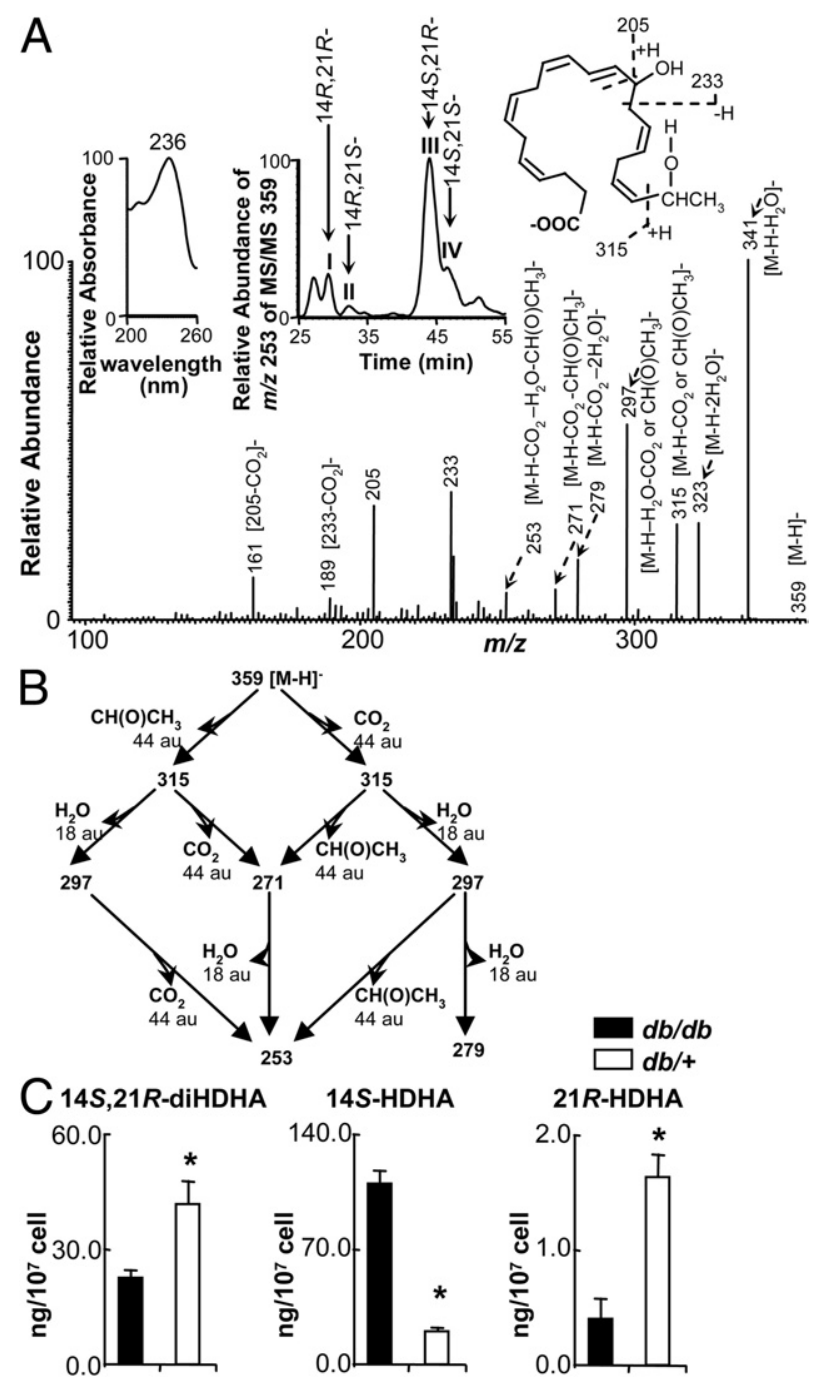

Figure 1. Differences between macrophages from diabetic $d b / d b$ and nondiabetic $d b /+$ mice in producing $14 S, 21 R$-diHDHA and its biosynthetic intermediates $14 S$-HDHA and 21R-diHDHA. Samples were analyzed by ChiralpakIA-based chiral LC-UV-MS/MS. A: $14 S, 21 R$-diHDHA identification (peak III) MS/MS spectrum, UV spectrum (top left), MS/MS-based structure elucidation (top right), and chiral LC chromatogram (top middle) with diastereomers at peak I, II, and IV as annotated. B: MS/MS fragmentation pathways for $14 S, 21 R$-diHDHA. C: Amount of $14 S, 21 R$-diHDHA, $14 S$-HDHA, or $21 R$-HDHA generated by macrophages (ng/10 $70^{7}$ cells). Data are reported as means \pm SEM $(n=3) .{ }^{*} P<0.05$.

There are four 14,21-diHDHA diastereomers resulting from four different combinations of $R / S$ configurations of $\mathrm{C} 14$ and $\mathrm{C} 21$ (14R,S and 21R,S). The chiral LC retention time of 14,21-diHDHA in peak III (Figure 1A, top middle) matched that of $14 S, 21 R$-diHDHA, which had different retention time than the other three 14,21-diHDHA diastereomers. ${ }^{16}$ Therefore, peak III is $14 S, 21 R$-diHDHA. The formation of $14 S, 21 R$-diHDHA was decreased in diabetic $d b / d b$ macrophages, compared with nondiabetic $d b /+$ macrophages (Figure 1C), based on the chiral LC-MS/MS quantification.

To obtain biochemical insights into the impairment of 14S,21R-diHDHA biosynthesis in diabetes, we studied the biosynthetic intermediates $14-\mathrm{HDHA}$ and $21-\mathrm{HDHA}$ in $d b / d b$ and $d b /+$ macrophages. ${ }^{8}$ A significantly higher level of $14 S-H D H A$ was generated by $d b / d b$ macro- 
A

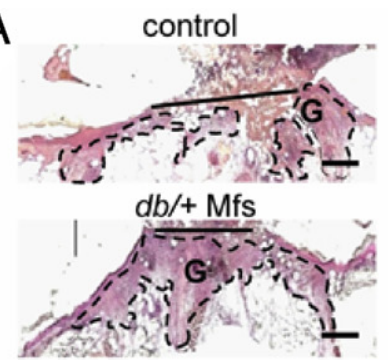

D

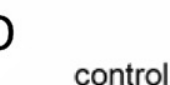

control
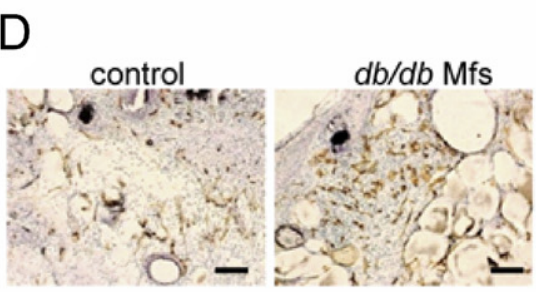

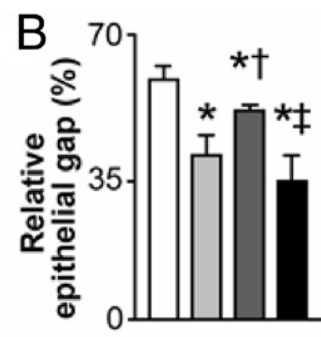

C

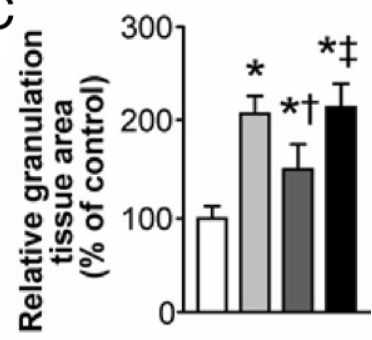

$14 S, 21 R$-diHDHA-

treated $d b / d b \mathrm{Mfs}$ $14 S, 21 R$-diHDHA-treated $d b / d b$ Mfs

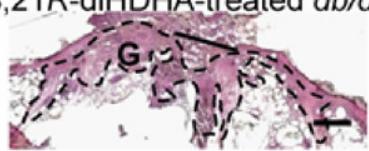

s vemon $\square$ control

E

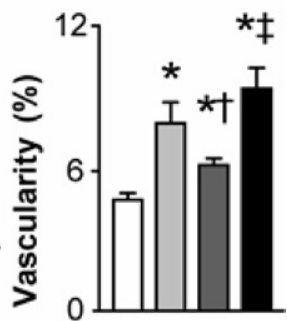

Figure 2. $14 S, 21 R$-diHDHA recovers impaired prohealing functions of $d b / d b$ macrophages (Mfs) in cutaneous wounds. Splinted excisional wounds were created in the skin of $d b / d b$ mice. At day 2 after wounding, $d b / d b$ macrophages, $14 S, 21 R$-diHDHA-treated $d b / d b$ macrophages, $d b /+$ macrophages (10 6 cells/wound), or RPMI 1640 vehicle control medium was administered to wounds. Wounded skin was analyzed at day 8 after wounding. A: Representative micrographs of H\&E-stained cryosections of wounds. A long black line marks the epithelial gap; dashed lines circumscribe areas of granulation tissue (G). B: Relative epithelial gap $(n=8)$. C: Relative granulation tissue area $(n=8)$. D: Representative microphotographs of CD31 ${ }^{+}$blood vessels in wounds. E: Quantification of wound vascularity $(n=18)$ at day 8 after wounding. Data are reported as means \pm SEM. ${ }^{*} P<0.05$ versus control; ${ }^{\dagger} P<0.05$ versus $d b /+$ macrophage treatment; ${ }^{\ddagger} P<$ 0.05 versus $d b / d b$ macrophage treatment. Scale bars, $500 \mu \mathrm{m}(\mathbf{A}) ; 100 \mu \mathrm{m}(\mathbf{D})$.

phages, compared with $d b /+$ macrophages (Figure 1C). This coincided with up-regulated expression of L-12-LOX (see Supplemental Figure S2A at http://ajp.amjpathol. org), which is involved in DHA 14S-hydroxylation. However, the 21R-HDHA level was significantly lower in $d b / d b$ macrophages than in $d b /+$ macrophages (Figure $1 C$ ), which suggests that diabetes may impede $14 S, 21 R$ diHDHA biosynthesis by interfering with 21-hydroxygenation. $^{8}$

We studied the 14S,21R-diHDHA biosynthesis in selected typical types of cells that are known to participate in wound healing. ${ }^{2}$ The results from our chiral LC-MS/MS analysis show that PMNs, platelets, and skin cells (including fibroblasts and DMVECs) did not produce 14S,21RdiHDHA; whereas lymphocytes ( $T$ cells and B cells) produced a much lesser amount of 14S,21R-diHDHA, compared with macrophages, and platelets produced the intermediate 14S-HDHA for the biosynthesis of 14S,21R-diHDHA (Figure 1A; see also Supplemental Figure S3 at http://ajp.amjpathol.org). These data suggest that macrophages produce 14S,21R-diHDHA in wounds, especially at days 2 to 4 after wounding, when macrophages are the major leukocytes in wounds. ${ }^{2}$ These results motivated us to further study the relation of decreased generation of $14 S, 21 R$-diHDHA to impaired prohealing functions of diabetic macrophages.

\section{S,21R-diHDHA Recovers Impaired \\ Prohealing Functions of Macrophages of Diabetic db/db Mice}

To determine whether the impaired prohealing functions of $d b / d b$ macrophages is associated with decreased 14S,21R-diHDHA generation, $d b /+, d b / d b$ macrophages and 14S,21R-diHDHA-treated $d b / d b$ macrophages were injected into wounds of $d b / d b$ mice at day 2 after wounding, when considerable numbers of endogenous macrophages are recruited in the natural course of wound healing. ${ }^{1,2}$ Compared with vehicle controls, the administration of $d b /+$ macrophages or $d b / d b$ macrophages significantly promoted re-epithelialization by decreasing the epithelial gap and increasing formation of granulation tissue (Figure 2, A-C). In addition, $d b / d b$ macrophages showed impaired functions in accelerating wound healing, compared with $d b /+$ macrophages (Figure 2, A-C), which is consistent with a previous report. ${ }^{4} 14 S, 21 R$ diHDHA-treated $d b / d b$ macrophages considerably decreased the epithelial gap and increased granulation tissue area, compared with $d b / d b$ macrophage treatment (Figure 2, A-C). These results provide evidence that reduced generation of $14 S, 21 R$-diHDHA in diabetic $d b / d b$ macrophages contributed to impaired prohealing functions, and that these functions were restored by $14 S, 21 R$ diHDHA treatment.

Impaired angiogenesis is a critical hallmark of nonhealing wounds in diabetes. ${ }^{1}$ Our data demonstrate that wounds of $d b / d b$ mice injected with $d b /+$ or $d b / d b$ macrophages exhibited increased vascularity, compared with wounds of control animals (Figure 2, D and E). In addition, significantly more CD $31^{+}$vessels were found in the wound beds injected with 14S,21R-diHDHA-treated $d b / d b$ macrophages than with untreated $d b / d b$ macrophages (Figure 2, D and E). Indeed, untreated $d b / d b$ macrophages were less efficient than $d b /+$ macrophages in promoting angiogenesis (Figure 2, D and E). Thus, 14S,21R-diHDHA counteracted diabetes impairment and recovered macrophage prohealing functions, including enhanced angiogenesis.

To further understand the cellular mechanism by which 14S,21R-diHDHA rescues impaired macrophage pro- 


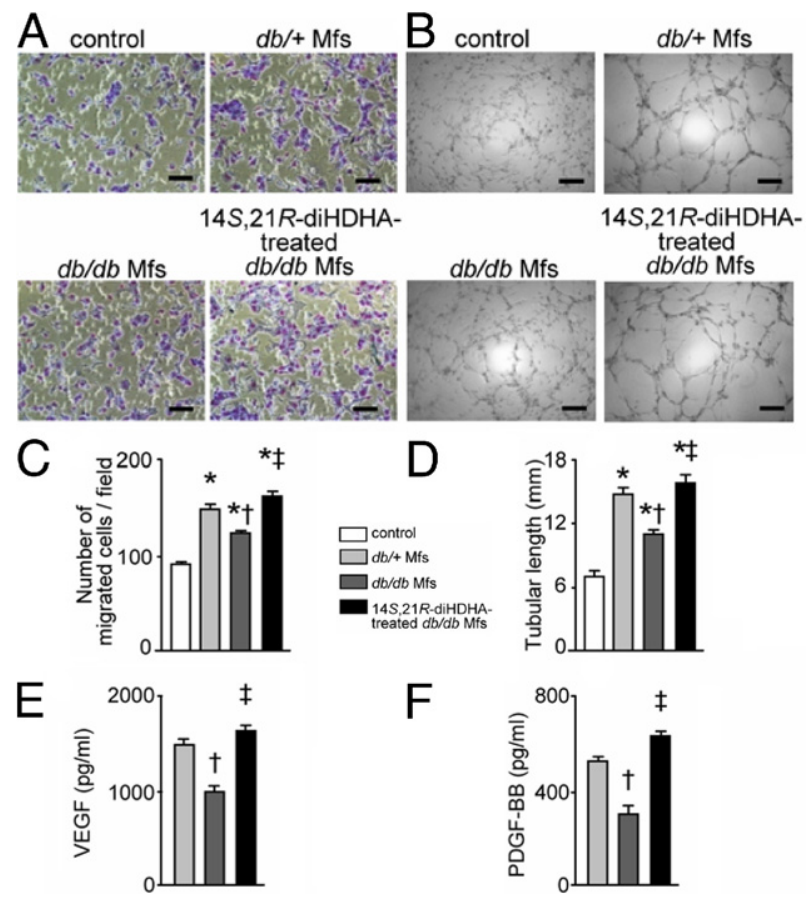

Figure 3. $14 S, 21 R$-diHDHA treatment restores $d b / d b$ macrophage function in cellular processes of angiogenesis in $d b / d b$ DMVECs. Representative micrographs of DMVEC migration (A) and vasculature formation (B) in RPMI 1640 vehicle control medium or in MCM. Data are quantified as migrated DMVECs per field $(n=15)(\mathbf{C})$ and as vasculature length per field $(n=4)$ (D). VEGF (E) and PGDF-BB (F) secreted from $d b / d b$ macrophages, $14 S, 21 R$ diHDHA-treated $d b / d b$ macrophages, or $d b /+$ macrophages were measured using a Bio-Plex protein array $(n=3)$. Scale bars $=100 \mu \mathrm{m}$. Data are reported as means \pm SEM. ${ }^{*} P<0.05$ versus control; ${ }^{\dagger} P<0.01$ versus $d b /+$ MCM; ${ }^{\ddagger} P<0.05$ versus $d b / d b$ MCM.

healing functions in diabetes, we studied diabetic $d b / d b$ DMVEC migration and vasculature formation in MCM. Media conditioned by $d b /+$ and $d b / d b$ macrophages considerably increased $d b / d b$ DMVEC migration and enhanced vasculature formation, compared with controls (non-MCM) (Figure 3, A-D). The $d b / d b$ MCM was significantly less effective in promoting $d b / d b$ DMVEC migration and vasculature formation, compared with $d b /+$ MCM, demonstrating that macrophage functions in angiogenesis were impaired in diabetic mice. When conditioned medium from 14S,21R-diHDHA-treated $d b / d b$ macrophages was used, $d b / d b$ DMVEC migration and vasculature formation was significantly enhanced, relative to $d b / d b$ MCM (Figure 3, A-D). These data indicate that 14S,21R-diHDHA enhanced functions of $d b / d b$ macrophages in angiogenesis through promoting DMVEC migration and vasculature formation.

Macrophages promote angiogenesis by secreting angiogenic cytokines such as VEGF and PDGF-BB. ${ }^{1,2,38}$ We postulated, therefore, that 14S,21R-diHDHA enhances the functions of $d b / d b$ macrophages in angiogenesis, in part, by increasing their angiogenic cytokine production. The results demonstrated that lesser amounts of VEGF and PDGF-BB were released by $d b / d b$ macrophages than by $d b /+$ macrophages (Figure 3, E and F), consistent with impaired functions of $d b / d b$ macrophages. This impairment was diminished by $14 S, 21 R$ diHDHA, which activated $d b / d b$ macrophages to secrete significantly more VEGF and PDGF-BB under simulated hyperglycemia (Figure 3, E and F). Taken together, these results suggest that $14 S, 21 R$-diHDHA treatment restored the impaired prohealing functions of $d b / d b$ macrophages in angiogenesis by enhancing $d b / d b$ macrophage paracrine capacity.

\section{2/15-LOX ${ }^{-1-}$ Macrophages Exhibit Impaired Prohealing Functions Associated with Decreased 14S,21R-diHDHA Biosynthesis}

To further confirm that endogenous 14S,21R-diHDHA positively regulated macrophage prohealing functions, 12/15-LOX ${ }^{-1-}$ macrophages were compared with wildtype C57BL/6J macrophages. We found that 12/15$\mathrm{LOX}^{-1-}$ macrophages did not produce 14S,21RdiHDHA, whereas macrophages from C57BL/6J, BALB/c, $d b / d b$, or $d b /+$ mice $d^{2} d^{8,16}$ (Figure $1 A$ ). This finding was expected, because 12/15-LOX is the only LOX available in macrophages that catalyzes the 14S-hydroperoxylation step ${ }^{39}$ in 14S,21R-diHDHA biosynthetic pathways and 12/15-LOX could not be detected in 12/15-LOX ${ }^{-1-}$ macrophages. After injection into wounds, C57BL/6J macrophages significantly promoted re-epithelialization with a decreased epithelial gap and promoted granulation tissue formation, as shown by an augmented granulation tissue area, compared with vehicle control (Figure 4. A-C). 12/15-LOX ${ }^{-1-}$ macrophages had no effect on re-epithelialization, but they did cause a slight increase in granulation tissue formation (Figure 4, A-C). Thus, 12/15$\mathrm{LOX}^{-1-}$ macrophages exhibited impaired functions in accelerating wound healing, compared with C57BL/6J macrophages (see Supplemental Figure S2 at http:// ajp.amjpathol.org). After treatment with 14S,21R-diHDHA, 12/15-LOX ${ }^{-1-}$ macrophages significantly accelerated wound healing. 14S,21R-diHDHA-treated 12/15-LOX ${ }^{-1-}$ macrophages decreased the epithelial gap and increased granulation tissue area, compared with untreated 12/15-LOX-1- macrophages (Figure 4, A-C).

The effect of decreased 14S,21R-diHDHA production on macrophage functions for angiogenesis was further addressed. We found that wounds injected with 12/15$\mathrm{LOX}^{-1-}$ macrophages did not exhibit increased vascularity, relative to the control, and also had much less vascularity relative to $\mathrm{C} 57 \mathrm{BL} / 6 \mathrm{~J}$ macrophage treatment (Figure 4, D and E). However, significantly higher wound vascularity was found in wounds injected with 14S,21R-diHDHA-treated 12/15-LOX ${ }^{-1-}$ macrophages than in wounds injected with untreated 12/15-LOX LI- $^{-1}$ macrophages (Figure 4, D and E). Thus, impaired prohealing functions of 12/15-LOX ${ }^{-1-}$ macrophages are associated with decreased biosynthesis of $14 S, 21 R$ diHDHA.

Given that i) macrophages are the main leukocytes, ii) there are relatively fewer PMNs in wounds at days 2 to 3 after wounding, and iii) macrophages produce 14S,21RdiHDHA, we wanted to clarify whether application of 14S,21R-diHDHA can ameliorate wound healing after macrophages become the dominant leukocytes. Wound healing in 12/15-LOX ${ }^{-1-}$ mice was compared 
A
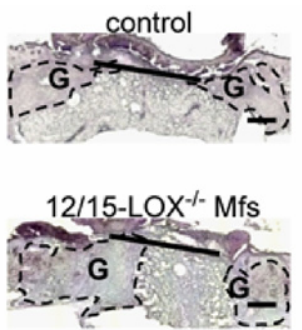

$\mathrm{D}$

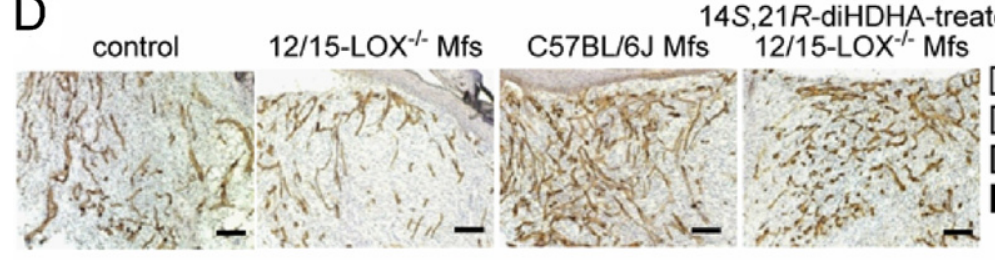

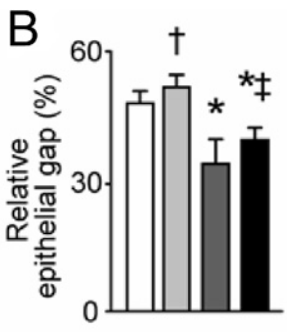

14S,21R-diHDHA-treated
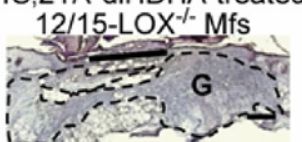

14S,21R-diHDHA-treated

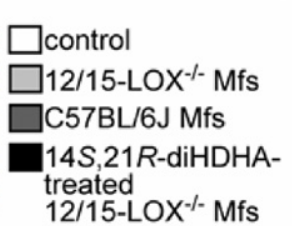

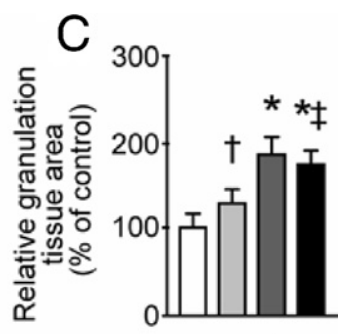

$\mathrm{E}$

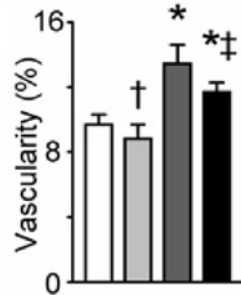

Figure 4. 14S,21R-diHDHA treatment restores macrophage prohealing functions impaired by 12/15-LOX deficiency. Splinted excisional wounds were created in the skin of C57BL/6J mice. Wounds were then treated with vehicle control, 12/15-LOX ${ }^{-/-}$macrophages, 14S,21R-diHDHA-treated $12 / 15$-LOX ${ }^{-/-}$macrophages, or C57BL/6J macrophages ( $10^{6}$ cells/wound) at day 2 after wounding. A: Representative micrographs of H\&E-stained cryosections of wounded skin collected at day 5 after wounding. A long black line marks the epithelial gap; dashed lines circumscribe areas of granulation tissue $(G)$. B: Relative epithelial gap $(n=8)$. C: Relative granulation tissue area $(n=8)$. D: Representative microphotographs of CD31 ${ }^{+}$blood vessels in wounds. E: Quantification of wound vascularity $(n=18)$ at day 7 after wounding. Data are reported as means \pm SEM. ${ }^{*} P<0.05$ versus control; ${ }^{\dagger} P<0.05$ versus $\mathrm{C} 57 \mathrm{BL} / 6 \mathrm{~J}$ macrophage treatment; ${ }^{\ddagger} P<0.05$ versus 12/15-LOX ${ }^{-1-}$ macrophage treatment. Scale bars, $500 \mu \mathrm{m}(\mathbf{A}) ; 100 \mu \mathrm{m}(\mathbf{D})$

with that in wild-type C57BL/6J mice. Previous data showed decreased 14S,21R-diHDHA formation in wounds of 12/15-LOX ${ }^{-1-}$ mice, compared with wounds of C57BL/6J mice. ${ }^{16}$ In addition, correlative with diminished formation of $14 S, 21 R$-diHDHA, histological analysis showed that 12/15-LOX ${ }^{-1-}$ mice exhibited impaired cutaneous wound healing, compared with C57BL/6J mice, as well as delayed re-epithelialization (Figure 5, A and $B$ ), decreased granulation tissue formation (Figure 5, A and C), and decreased vascularity (Figure 5, D and E). Application of 14S,21R-diHDHA into wounds of 12/15-LOX ${ }^{-1-}$ mice at day 2 after wounding significantly promoted re-epithelialization and granulation tissue formation, as well as vascularity (Figure 5). These data suggest that $14 S, 21 R$-diHDHA may ame- liorate wound healing through modulating macrophage functions.

\section{S,21R-diHDHA Reduces Generation of ROS and Increases IL-10 Expression in $\mathrm{db} / \mathrm{db}$ Macrophages}

Oxidative stress is a critical cause for impaired macrophage functions in diabetes. ${ }^{18,19}$ We therefore investigated whether 14S,21R-diHDHA treatment reduces ROS generation. A high concentration of glucose (25 mmol/L) induced ROS generation in $d b /+$ macrophages. A low glucose concentration $(5.5 \mathrm{mmol} / \mathrm{L})$ resulted in slightly decreased ROS generation in $d b / d b$ macrophages

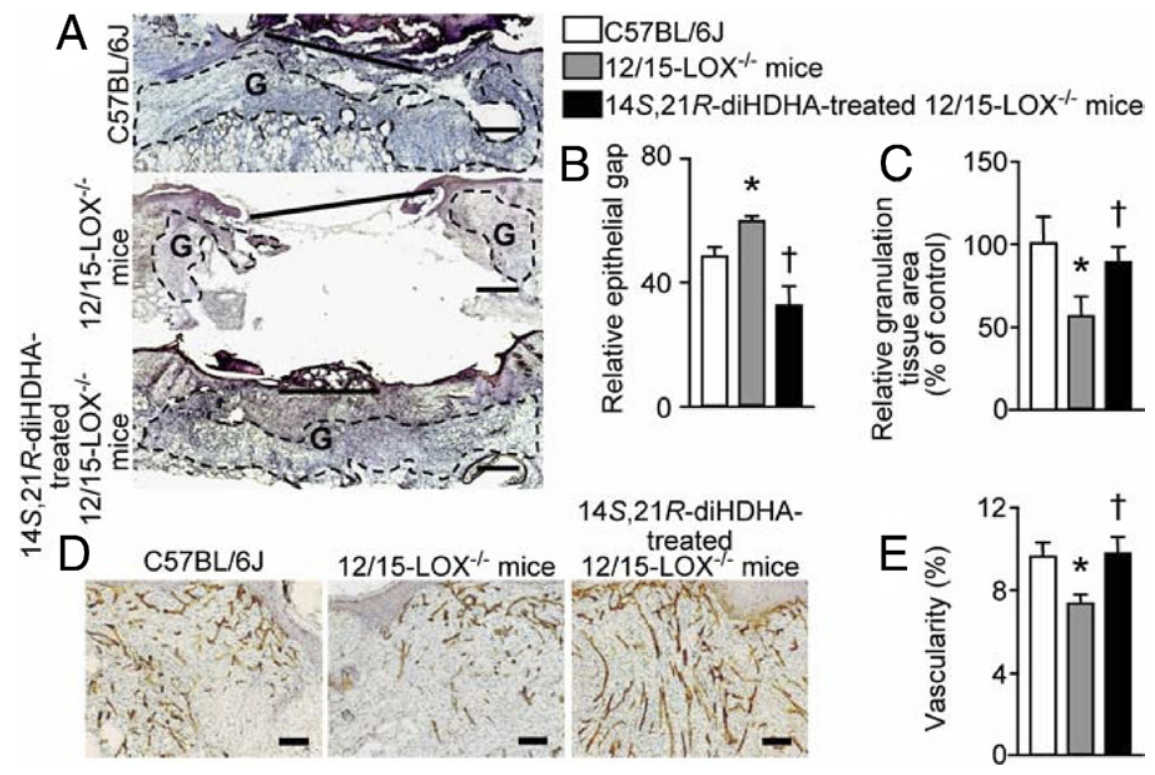

Figure 5. $14 S, 21 R$-diHDHA ameliorates impaired cutaneous wound healing in $12 / 15$ $\mathrm{LOX}^{-/-}$mice. Splinted excisional wounds were made in the skin of $12 / 15-\mathrm{LOX}^{-1-}$ mice and C57BL/6J mice. Wounds were then treated with $14 S, 21 R$-diHDHA (50 ng/wound) at day 2 after wounding. Wounds of $\mathrm{C} 57 \mathrm{BL} / 6 \mathrm{~J}$ mice received only vehicle. A: Representative micrographs of H\&E-stained cryosections of wounds at day 5 after wounding. A long black line marks the epithelial gap; dashed lines circumscribe areas of granulation tissue $(G)$. B: Relative epithelial gap $(n=8)$. C: Relative granulation tissue area $(n=8)$. D: Representative microphotographs of CD $31^{+}$blood vessels in wounds. E: Quantification of wound vascularity $(n=18)$ at day 7 after wounding. Data are reported as means \pm SEM. ${ }^{*} P<0.05$ versus $\mathrm{C} 57 \mathrm{BL} / 6 \mathrm{~J}$ mice; ${ }^{\dagger} P<0.05 \mathrm{com}-$ pared $12 / 15-\mathrm{LOX}^{-/-}$mice treated with vehicle control. Scale bars, $500 \mu \mathrm{m}$ (A); $100 \mu \mathrm{m}$ (D). 
A

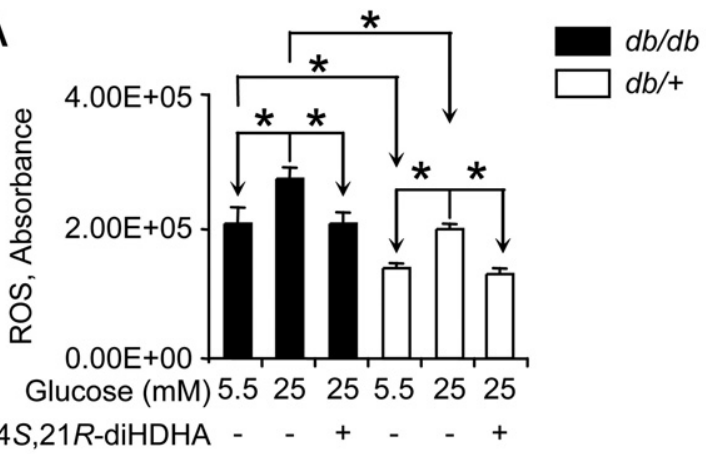

B
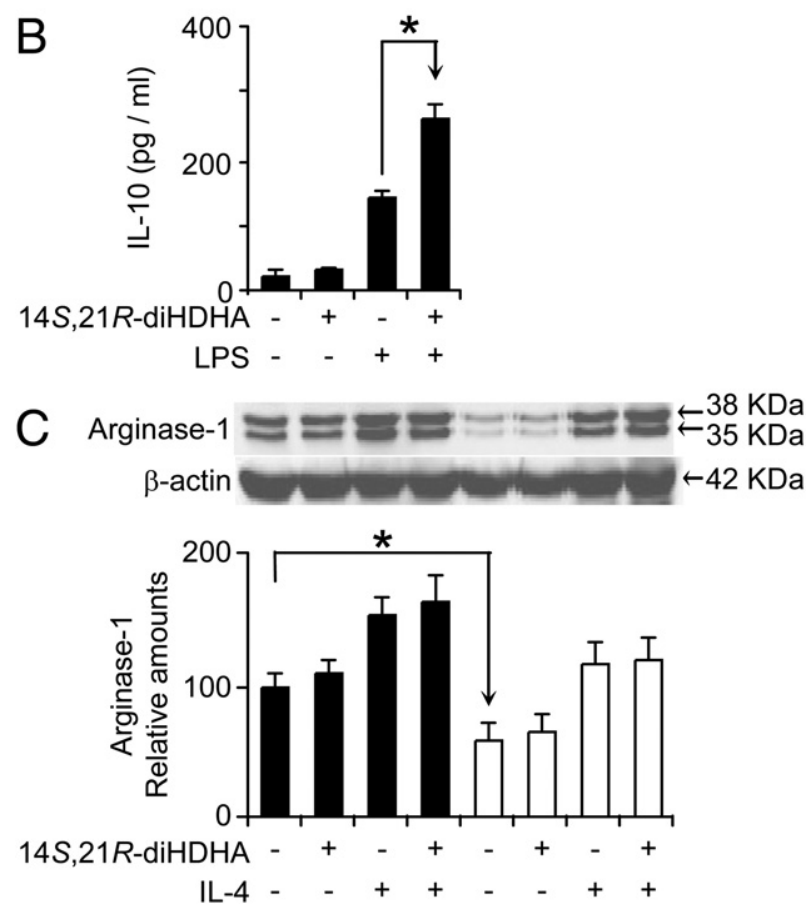

Figure 6. $14 S, 21 R$-diHDHA reduces ROS generation induced by high concentrations of glucose and increases IL-10 expression in $d b / d b$ macrophages under LPS stimulation. A: ROS generation. $d b / d b$ and $d b /+$ macrophages were cultured in $5.5 \mathrm{mmol} / \mathrm{L}$ or $25 \mathrm{mmol} / \mathrm{L}$ glucose and treated with $14 S, 21$ diHDHA. ROS generation in cells was assessed using the probe DCFH-DA $(n=4)$. B: IL-10 expression. IL-10 secreted from $d b / d b$ macrophages treated with $14 S, 21 R$-diHDHA, LPS, or $14 S, 21 R$-diHDHA plus LPS was measured using an IL-10 enzyme-linked immunosorbent assay kit $(n=3)$. C: Arginase-1 expression. Quiescent $d b / d b$ and $d b /+$ macrophages were cultured for 24 hours in RPMI 1640 medium with $14 S, 21 R$-diHDHA, IL-4, or both IL-4 and $14 S, 21 R$-diHDHA. Macrophage lysates were analyzed by Western blot. Arginase- 1 quantities relative to $\beta$-actin were measured based on optical densities of the gel bands and were normalized to that of nontreated $d b / d b$ macrophages $(n=3) .14 S, 21 R$-diHDHA, $100 \mathrm{nmol} / \mathrm{L} ; \mathrm{IL}-4,10 \mathrm{ng} / \mathrm{mL} ; \mathrm{LPS}$, $100 \mathrm{ng} / \mathrm{mL}$. Data are reported as means \pm SEM. ${ }^{*} P<0.05$

(Figure $6 \mathrm{~A}$ ). The $d b / d b$ macrophages produced more ROS, compared with $d b /+$ macrophages (Figure 6A). 14S,21R-diHDHA treatment decreased ROS generation by $d b /+$ and $d b / d b$ macrophages in high-glucose conditions (Figure 6A). Thus, 14S,21R-diHDHA may affect macrophage function by reducing ROS generation under high-glucose conditions.

$\mathrm{IL}-10$ is an important anti-inflammatory cytokine upregulated by LPS. ${ }^{29}$ LPS stimulation resembles the potential of infection to wounds. To determine the effect of 14S,21R-diHDHA on IL-10 expression, we treated $d b / d b$ macrophages with 14S,21R-diHDHA alone, 14S,21R-
diHDHA plus LPS, LPS alone, or vehicle control. We found that $14 S, 21 R$-diHDHA significantly promoted LPSinduced IL-10 generation by macrophages, although 14S,21R-diHDHA alone did not affect IL-10 generation by $\mathrm{db} / \mathrm{db}$ macrophages (Figure 6B). These results indicate that $14 S, 21 R$-diHDHA enhanced the anti-inflammatory functions of $d b / d b$ macrophages. We also assessed the effect of 14S,21R-diHDHA on IL-4-induced alternative activation, ${ }^{40}$ which results in a prohealing phenotype in macrophages and characteristically involves arginase-1 expression. 14S,21R-diHDHA did not affect this type of activation of macrophages under the in vitro conditions used in the present study (Figure 6C).

\section{Discussion}

Wound healing is a dynamic process that is characterized by inflammation, tissue regeneration, and remodeling. ${ }^{2}$ We have observed that wounded skin can generate $14 S, 21 R$-diHDHA. ${ }^{8,16}$ Several kinds of cells contribute to wound healing. ${ }^{2}$ Our data show that macrophages possess a greater capability to produce 14S,21R-diHDHA, compared with several other typical cell types usually present in wounds. 14S,21R-diHDHA formation was reduced in diabetic $d b / d b$ macrophages, compared with nondiabetic $d b /+$ macrophages (Figure 1), which suggests that diabetes dysregulation of macrophage biosynthesis of $14 S, 21 R$-diHDHA could contribute to diabetes impairment of $14 S, 21 R$-diHDHA production in diabetic wounds. ${ }^{16}$ The formation of $14 S-\mathrm{HDHA}$, a marker of 12 LOX activity, ${ }^{8}$ was elevated in diabetic wounds. Nevertheless, the decreased 21R-HDHA formation (Figure 1) suggests that diabetes attenuates 21R-hydroxylation, which could contribute to reduced 14S,21R-diHDHA generation. The $21 R$-hydroxylation is likely to be catalyzed by a specific $\mathrm{P} 450^{41}$ in macrophages, ${ }^{8,42}$ which is of interest for our future studies.

Diabetic hyperglycemia and accompanying oxidative stress might affect biosynthesis of $14 S, 21 R$-diHDHA by altering P450 or disrupting the transfer of biosynthetic intermediates ${ }^{8}$ between 12-LOX and P450 within or between cells. 12/15-LOX ${ }^{-1-}$ macrophages are deficient in 14S,21R-diHDHA generation; we therefore used 12/15$\mathrm{LOX}^{-1-}$ macrophages as negative control to study the role of this autacoid-type mediator in macrophage functions. Although platelets are unable to produce 14S,21R$\operatorname{diHDHA}$, and lymphocytes barely produce this mediator, both cell types produce the intermediate 14S-HDHA of 14S,21R-diHDHA biosynthesis (see Supplemental Figure S3 at $h$ ttp://ajp.amjpathol.org). The 14S-HDHA produced by platelets or lymphocytes was likely transported to macrophages and there converted to $14 S, 21 R$-diHDHA through the transcellular biosynthesis mechanism for lipid mediators. ${ }^{43,44}$

Macrophages are the main leukocytes in wounds at days 2 to 3 after wounding, when the wounds are still in the inflammation phase. ${ }^{2}$ They are activated to phagocytose apoptotic PMNs, debris, and bacteria, and then coordinate later events of healing, including re-epithelialization, granulation tissue formation, and angiogenesis. 


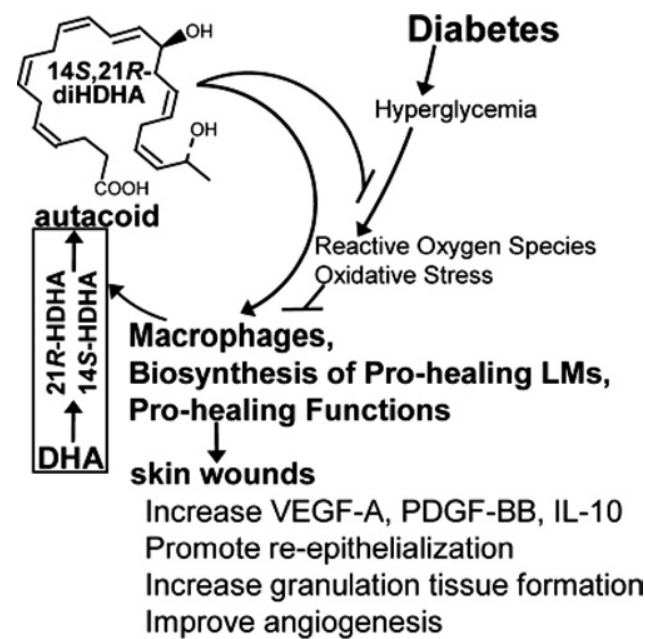

Figure 7. Impairment of prohealing functions of diabetic $d b / d b$ macrophages is associated with reduced $14 S, 21 R$-diHDHA formation. Diabetes decreases the generation of $14 S, 21 R$-diHDHA by macrophages. Treatment with $14 S, 21 R$-diHDHA rescues diabetes-impaired macrophage promotion of wound re-epithelialization, granulation tissue formation, and vascularization The mechanism of $14 S, 21 R$-diHDHA action on macrophages includes reducing ROS generation under hyperglycemia, increasing IL-10 expression, increasing the secretion of VEGF and PDGF-BB, and enhancing the macrophage promotion of endothelial cell migration and vasculature formation.

Impaired macrophage functions are among the crucial defects responsible for delayed wound healing in diabetics. Compared with normal macrophages, diabetic macrophages display detrimental pathophysiological responses, such as expressing low levels of angiogenic cytokines, ${ }^{4,17-19}$ including reduced expression of VEGF and PDGF-BB (Figure 3). Moreover, the association of decreased formation of $14 S, 21 R$-diHDHA with impaired prohealing functions of $d b / d b$ macrophages indicates that impaired functions of diabetic macrophages also includes impaired formation of this autacoid. This association was further confirmed by the fact that 12/15$\mathrm{LOX}^{-1-}$ macrophages could not produce $14 S, 21 R$ diHDHA and exhibited attenuated promotion of healing that could be restored by $14 S, 21 R$-diHDHA treatment (Figure 4). Impaired functions of 12/15-LOX ${ }^{-1-}$ macrophages may not only result from halted $14 S, 21 R$-diHDHA formation, but may also stem from other detrimental effects of 12/15-LOX deletion; however, 14S,21R-diHDHA treatment can restore macrophage functions, indicating that $14 S, 21 R$-diHDHA can redeem those effects. Additionally, 12/15-LOX ${ }^{-1-}$ mice exhibiting delayed wound healing. The application of $14 S, 21 R$-diHDHA at day 2 after wounding, when macrophages significantly infiltrate into wounds, counteract such impairment (Figure 5). This further suggests the reparative roles of $14 S, 21 R$-diHDHA via action on macrophages in wounds. However, the paracrinic action of macrophage 14S,21R-diHDHA on other kinds of cells in wounds cannot be excluded and should be investigated in future studies.

During the inflammatory phase of wound repair, ROS generated by PMNs and macrophages destroy harmful microorganisms and stimulate expression of some growth factors. ${ }^{45}$ However, overproduction of ROS leads to excessive oxidative stress and results in cellular dysfunction and functional alterations of DNA, lipids, and proteins. ${ }^{17,45,46}$ The high glucose concentration in diabetic hyperglycemia is a critical initiating factor for ROS generation. ${ }^{47}$ In this regard, $d b / d b$ macrophages generated much more ROS than $d b /+$ macrophages, which is consistent with a previous report. ${ }^{18}$ High glucose concentrations induce significant increases in ROS production in macrophages (Figure 6). Thus, diabetic hyperglycemia might stimulate macrophages to produce excessive ROS, which cause macrophage dysfunction. Moreover, the suppression of oxidative stress has been shown to reduce the abnormally high levels of proinflammatory cytokines in diabetic macrophages. ${ }^{19} 14 S, 21 R$ diHDHA was found to decrease ROS generation (Figure 6 ), providing another possible means by which $14 S, 21 R$ diHDHA treatment mediates recovery of macrophage prohealing functions in diabetes.

Heterogeneity of macrophages has recently been proposed. Alternatively activated macrophages, as distinct from classically activated macrophages, promote wound healing, and macrophages are capable of switching phenotypes under different stimuli. ${ }^{40}$ However, 14S,21RdiHDHA did not affect arginase-1 expression under IL-4 stimulus (Figure 6), a property of alternatively activated macrophages, suggesting that $14 S, 21 R$-diHDHA does not act on the macrophage switching to an alternative phenotype. 14S,21R-diHDHA increased IL-10 expression by $d b / d b$ macrophages under LPS stimulation (Figure 6), suggesting that the recovery of diabetic prohealing functions by $14 S, 21 R$-diHDHA may involve augmenting IL-10 generation. Of note, $d b / d b$ macrophages expressed more arginase-1, compared with $d b /+$ macrophages (Figure 6). Arginase-1 effectively competed with nitric oxide synthase for arginine and converted arginine to urea and ornithine, thus preventing the overproduction of nitric oxide. ${ }^{48}$ Increased expression of arginase- 1 and IL-10 in $d b / d b$ macrophages, compared with $d b /+$ macrophages, may be an adaptive response under the stress of diabetes. ${ }^{49}$

The DHA-derived lipid mediator 14S,21R-diHDHA acts as an macrophage-produced autacoid that promotes the prohealing functions of macrophages (Figure 7). Reduced formation of $14 S, 21 R$-diHDHA in $d b / d b$ macrophages is associated with their impaired prohealing functions. 14S,21R-diHDHA treatment of macrophages reduced ROS generation, promoted IL-10 generation, and enhanced diabetic macrophage prohealing functions. These observations provide mechanistic insights into wound healing and point toward possible treatment of wounds in diabetic patients using autologous macrophages treated with $14 S, 21 R$-diHDHA or related compounds.

\section{Acknowledgments}

We thank Drs. Nicolas G. Bazan and Haydee E.P. Bazan (Neuroscience Center of Excellence at the Louisiana State University Health Sciences Center, New Orleans, LA) for 12/15-LOX knockout mice and also for helpful advice. We thank Dr. Eric C.B. Milner for excellent editing 
and Dr. Qiang Wu and Jose A. Galindo for technical assistance.

\section{References}

1. Falanga V: Wound healing and its impairment in the diabetic foot Lancet 2005, 366:1736-1743

2. Broughton $\mathrm{G}$ 2nd, Janis JE, Attinger CE: The basic science of wound healing. Plast Reconstr Surg 2006, 117:12S-34S

3. Danon D, Kowatch MA, Roth GS: Promotion of wound repair in old mice by local injection of macrophages. Proc Natl Acad Sci USA 1989, 86:2018-2020

4. Maruyama K, Asai J, li M, Thorne T, Losordo DW, D'Amore PA: Decreased macrophage number and activation lead to reduced lymphatic vessel formation and contribute to impaired diabetic wound healing. Am J Pathol 2007, 170:1178-1191

5. Lucas T, Waisman A, Ranjan R, Roes J, Krieg T, Müller W, Roers A, Eming SA: Differential roles of macrophages in diverse phases of skin repair. J Immunol 2010, 184:3964-3977

6. Mirza R, DiPietro LA, Koh TJ: Selective and specific macrophage ablation is detrimental to wound healing in mice. Am J Pathol 2009, 175:2454-2462

7. Khanna S, Biswas S, Shang Y, Collard E, Azad A, Kauh C, Bhasker V, Gordillo GM, Sen CK, Roy S: Macrophage dysfunction impairs resolution of inflammation in the wounds of diabetic mice. PLoS One 2010, 5:e9539

8. Lu Y, Tian H, Hong S: Novel 14,21-dihydroxy-docosahexaenoic acids: structures, formation pathways, and enhancement of wound healing. J Lipid Res 2010, 51:923-932

9. Abbatecola AM, Evans W, Paolisso G: PUFA supplements and type 2 diabetes in the elderly. Curr Pharm Des 2009, 15:4126-4134

10. Nettleton JA, Katz R: n-3 Long-chain polyunsaturated fatty acids in type 2 diabetes: a review. J Am Diet Assoc 2005, 105:428-440

11. Montori VM, Farmer A, Wollan PC, Dinneen SF: Fish oil supplementation in type 2 diabetes: a quantitative systematic review. Diabetes Care 2000, 23:1407-1415

12. Shingel KI, Faure MP, Azoulay L, Roberge C, Deckelbaum RJ: Solid emulsion gel as a vehicle for delivery of polyunsaturated fatty acids: implications for tissue repair, dermal angiogenesis and wound healing. J Tissue Eng Regen Med 2008, 2:383-393

13. Serhan CN, Yang R, Martinod K, Kasuga K, Pillai PS, Porter TF, Oh SF, Spite M: Maresins: novel macrophage mediators with potent antiinflammatory and proresolving actions. J Exp Med 2009, 206:15-23

14. Hong S, Gronert K, Devchand PR, Moussignac RL, Serhan CN: Nove docosatrienes and 17S-resolvins generated from docosahexaenoic acid in murine brain, human blood, and glial cells. Autacoids in anti-inflammation. J Biol Chem 2003, 278:14677-14687

15. Gronert K, Maheshwari N, Khan N, Hassan IR, Dunn M, Laniado Schwartzman M: A role for the mouse 12/15-lipoxygenase pathway in promoting epithelial wound healing and host defense. J Biol Chem 2005, 280:15267-15278

16. Tian H, Lu Y, Shah SP, Hong S: 14S,21R-Dihydroxydocosahexaenoic acid remedies impaired healing and mesenchymal stem cell functions in diabetic wounds. J Biol Chem 2011, 286:4443-4453

17. Brownlee M: Biochemistry and molecular cell biology of diabetic complications. Nature 2001, 414:813-820

18. Ptak W, Klimek M, Bryniarski K, Ptak M, Majcher P: Macrophage function in alloxan diabetic mice: expression of adhesion molecules, generation of monokines and oxygen and $\mathrm{NO}$ radicals. Clin Exp Immunol 1998, 114:13-18

19. Haskins K, Bradley B, Powers K, Fadok V, Flores S, Ling X, Pugazhenthi S, Reusch J, Kench J: Oxidative stress in type 1 diabetes. Ann NY Acad Sci 2003, 1005:43-54

20. Tian H, Lu Y, Sherwood AM, Hongqian D, Hong S: Resolvins E1 and D1 in choroid-retinal endothelial cells and leukocytes: biosynthesis and mechanisms of anti-inflammatory actions. Invest Ophthalmol Vis Sci 2009, 50:3613-3620

21. Dona M, Fredman G, Schwab JM, Chiang N, Arita M, Goodarzi A, Cheng G, von Andrian UH, Serhan CN: Resolvin E1, an EPA-derived mediator in whole blood, selectively counterregulates leukocytes and platelets. Blood 2008, 112:848-855
22. Serhan CN, Gotlinger K, Hong S, Lu Y, Siegelman J, Baer T, Yang R, Colgan SP, Petasis NA: Anti-inflammatory actions of neuroprotectin D1/protectin D1 and its natural stereoisomers: assignments of dihydroxy-containing docosatrienes [Erratum appeared in $\mathrm{J}$ Immunol 2006, 176:3843]. J Immunol 2006, 176:1848-1859

23. Marcheselli VL, Hong S, Lukiw WJ, Tian XH, Gronert K, Musto A, Hardy M, Gimenez JM, Chiang N, Serhan CN, Bazan NG: Novel docosanoids inhibit brain ischemia-reperfusion-mediated leukocyte infiltration and pro-inflammatory gene expression [Erratum appeared in J Biol Chem 2003, 278:51974]. J Biol Chem 2003, 278:4380743817

24. Basselin M, Kim HW, Chen M, Ma K, Rapoport SI, Murphy RC, Farias SE: Lithium modifies brain arachidonic and docosahexaenoic metabolism in rat lipopolysaccharide model of neuroinflammation. J Lipid Res 2010, 51:1049-1056

25. Yang $P$, Chan D, Felix E, Madden T, Klein RD, Shureiqi I, Chen X, Dannenberg AJ, Newman RA: Determination of endogenous tissue inflammation profiles by LC/MS/MS: COX-and LOX-derived bioactive lipids. Prostaglandins Leukot Essent Fatty Acids 2006, 75:385-395

26. Gronert K, Clish CB, Romano M, Serhan CN: Transcellular regulation of eicosanoid biosynthesis. Methods Mol Biol 1999, 120:119-144

27. Bazan NG, Marcheselli VL, Lu Y, Hong S, Jackson F: Lipidomic approaches to neuroprotection signaling in the retinal pigment epithelium. Signal Transduction in the Retina. Edited by SJ Fliesler, OG Kisselev. Boca Raton, FL, CRC Press, 2008, pp 349-374

28. Hall LM, Murphy RC: Electrospray mass spectrometric analysis of 5-hydroperoxy and 5-hydroxyeicosatetraenoic acids generated by lipid peroxidation of red blood cell ghost phospholipids. J Am Soc Mass Spectrom 1998, 9:527-532

29. Barsness KA, Bensard DD, Partrick DA, Calkins CM, Hendrickson RJ, Mclntyre RC Jr: Endotoxin induces an exaggerated interleukin-10 response in peritoneal macrophages of children compared with adults. J Pediatr Surg 2004, 39:912-915; discussion 912-915

30. Galiano RD, Michaels J 5th, Dobryansky M, Levine JP, Gurtner GC: Quantitative and reproducible murine model of excisional wound healing. Wound Repair Regen 2004, 12:485-492

31. Michaels J 5th, Churgin SS, Blechman KM, Greives MR, Aarabi S, Galiano RD, Gurtner GC: db/db mice exhibit severe wound-healing impairments compared with other murine diabetic strains in a silicone-splinted excisional wound model. Wound Repair Regen 2007 15:665-670

32. Tian H, Lu Y, Shah SP, Hong S: Novel 14S,21-dihydroxy-docosahexaenoic acid rescues wound healing and associated angiogenesis impaired by acute ethanol intoxication/exposure. J Cell Biochem 2010, 111:266-273

33. Stepanovic V, Awad O, Jiao C, Dunnwald M, Schatteman GC: Leprdb diabetic mouse bone marrow cells inhibit skin wound vascularization but promote wound healing. Circ Res 2003, 92:1247-1253

34. Gee E, Milkiewicz M, Haas TL: p38 MAPK activity is stimulated by vascular endothelial growth factor receptor 2 activation and is essential for shear stress-induced angiogenesis. J Cell Physiol 2010, 222 $120-126$

35. Sharma GD, HE J, Bazan HE: p38 and ERK1/2 coordinate cellular migration and proliferation in epithelial wound healing: evidence of cross-talk activation between MAP kinase cascades. J Biol Chem 2003, 278:21989-21997

36. Louis CA, Mody V, Henry WL Jr, Reichner JS, Albina JE: Regulation of arginase isoforms I and II by IL-4 in cultured murine peritoneal macrophages. Am J Physiol 1999, 276:R237-R242

37. Ali MH, Schlidt SA, Chandel NS, Hynes KL, Schumacker PT, Gewertz $\mathrm{BL}$ : Endothelial permeability and IL-6 production during hypoxia: role of ROS in signal transduction. Am J Physiol 1999, 277:L1057-L1065

38. Pang C, Gao Z, Yin J, Zhang J, Jia W, Ye J: Macrophage infiltration into adipose tissue may promote angiogenesis for adipose tissue remodeling in obesity. Am J Physiol Endocrinol Metab 2008, 295: E313-E322

39. Siebert M, Krieg P, Lehmann WD, Marks F, Fürstenberger G: Enzymic characterization of epidermis-derived 12-lipoxygenase isoenzymes. Biochem J 2001, 355:97-104

40. Stout RD, Suttles J: Functional plasticity of macrophages: reversible adaptation to changing microenvironments. J Leukoc Biol 2004, 76: 509-513 
41. VanRollins M, Baker RC, Sprecher HW, Murphy RC: Oxidation of docosahexaenoic acid by rat liver microsomes. J Biol Chem 1984 259:5776-5783

42. Christmas P, Tolentino K, Primo V, Berry KZ, Murphy RC, Chen M, Lee DM, Soberman RJ: Cytochrome P-450 4F18 is the leukotriene B4 omega-1/omega-2 hydroxylase in mouse polymorphonuclear leukocytes: identification as the functional orthologue of human polymorphonuclear leukocyte CYP4F3A in the down-regulation of responses to LTB4. J Biol Chem 2006, 281:7189-7196

43. Serhan CN, Chiang N, Van Dyke TE: Resolving inflammation: dua anti-inflammatory and pro-resolution lipid mediators. Nat Rev Immunol 2008, 8:349-361

44. Farias SE, Zarini S, Precht T, Murphy RC, Heidenreich KA: Transcellular biosynthesis of cysteinyl leukotrienes in rat neuronal and glial cells. J Neurochem 2007, 103:1310-1318

45. Kapoor M, Kojima F, Appleton I, Kawai S, Crofford LJ: Major enzymatic pathways in dermal wound healing: current understand- ing and future therapeutic targets. Curr Opin Investig Drugs 2006, 7:418-422

46. Kämpfer H, Schmidt R, Geisslinger G, Pfeilschifter J, Frank S: Wound inflammation in diabetic ob/ob mice: functional coupling of prostaglandin biosynthesis to cyclooxygenase- 1 activity in diabetes-impaired wound healing. Diabetes 2005, 54:1543-1551

47. Wright E Jr, Scism-Bacon JL, Glass LC: Oxidative stress in type 2 diabetes: the role of fasting and postprandial glycaemia. Int $\mathrm{J}$ Clin Pract 2006, 60:308-314

48. Cederbaum SD, Yu H, Grody WW, Kern RM, Yoo P, Iyer RK: Arginases I and II: do their functions overlap? Mol Genet Metab 2004, 81 Suppl 1:S38-S44

49. Li SL, Reddy MA, Cai Q, Meng L, Yuan H, Lanting L, Natarajan R: Enhanced proatherogenic responses in macrophages and vascular smooth muscle cells derived from diabetic $\mathrm{db} / \mathrm{db}$ mice. Diabetes 2006, 55:2611-2619 